\title{
Audit of Intrapartum Care Based on the National Guideline for Midwifery and Birth Services
}

\section{Sonia Minooee ${ }^{1 \oplus}$, Masoumeh Simbar $^{2}$, Zohreh Sheikhan ${ }^{1}$ and Hamid Alavi Majd ${ }^{3}$}

\begin{abstract}
Providing high-quality maternity care is a worldwide health concern that necessitates regular assessment of intrapartum practice. In an observational study, we aimed to audit intrapartum care based on the National Guideline for Midwifery and Birth Services. Using quota sampling, a total of 200 pregnant women, admitted for normal vaginal delivery, were recruited from four educational hospitals in Tehran, Iran. An observational checklist was
\end{abstract}

\footnotetext{
'Midwifery and Reproductive Health Research Center, School of Nursing and Midwifery, Shahid Beheshti University of Medical Sciences, Tehran, Iran

${ }^{2}$ Department of Midwifery and Reproductive Health, Midwifery and Reproductive Health Research Center, School of Nursing and Midwifery, Shahid Beheshti University of Medical Sciences, Tehran, Iran

${ }^{3}$ Faculty of Paramedics, Department of Biostatistics, Shahid Beheshti University of Medical Sciences, Tehran, Iran
} 
Corresponding Author:

Masoumeh Simbar, Department of Midwifery and Reproductive Health, Midwifery and Reproductive Health Research Center, School of Nursing and Midwifery, Shahid Beheshti University of Medical Sciences, Cross of Vali-Asr and Neiaiesh Highway, Opposite to Rajaee Heart Hospital, Tehran, Iran.

Email:msimbar@yahoo.ac.ir 
developed based on the national guideline to assess the quality of provided care. Content and face validity of the tool were checked and confirmed. Reliability of the observational checklist and questionnaire was confirmed using concurrent observation (intrarater reliability; $r^{1 / 4} .93$ ) and test-retest $(r 1 / 4$.9) methods, respectively. We found that the compatibility of intrapartum care and the national guideline in different domains were as follows: history taking $88.3 \%$, vital sign measurement $64.6 \%$, performing Leopold's maneuver $38.5 \%$, initial assessment $83.4 \%$, labor care $22.5 \%$, using pain relief methods $63.5 \%$, labor progress assessment $71.5 \%$, process of delivery $89.5 \%$, and postpartum management $89.5 \%$. The findings indicate that additional attention and monitoring are required to align current intrapartum care practices with the national guidelines.

\section{Keywords}

audit, intrapartum care, labor, delivery, quality

Ensuring high-quality intrapartum care is a necessity in reducing maternal morbidity and mortality, particularly in developing countries (World Health Organization [WHO], 2014). Over the past 20 years, the number of women dying of obstetric complications has halved, and the global trend in maternal mortality indicates that the number of maternal deaths dropped from more than 543,000 to 287,000 per year (47\% decline) from 1990 to 2010 (United Nations Population Fund [UNPA], 2012), of which a majority of them can be prevented through improving the quality of care (WHO, 2007).

Ten countries have already reached the Millennium Development Goal target of a $75 \%$ reduction in maternal death of which Iran is one of them (UNPA, 2012). Based on the WHO $(2010,2014)$ reports, Iran could decrease maternal mortality up to $81 \%$ and $72 \%$, respectively. Over one million Iranian women give birth annually, of whom around $90 \%$ receive maternity care in hospitals (Akbary, 2005); therefore, providing safe and high-quality care can lead to considerable improvements in national rates of maternal morbidity and mortality.

Evidence indicates that certain intrapartum procedures such as routine episiotomy (Jiang, Qian, Carroli, \& Garner, 2017), routine restriction of oral intake during labor (Singata, Tranmer, \& Gyte, 2013), and routine vaginal examinations (Downe, Gyte, Dahlen, \& Singata, 2013) may be distressing or even harmful for some mothers (Lothian, 2009). Several of 
these procedures have been identified as costly practices that do not improve obstetric outcomes, whereas some are frequently performed in hospitals. To date, several studies have evaluated peripartum care in Iran (Changaee, Simbar, Irajpour, \& Akbari, 2014; Ganji, 2014; Moosavisadat, Lamyian, Parsap, \& Hajizadeh, 2011; Simbar, Dibazari, Saeidi, \& Alavi Majd, 2005; Simbar, Ghafari, Tork Zahrani, \& Alavi Majd, 2009; Simbar, Nahidi, Dolatian, \& Akbarzadeh, 2012); however, a few of them have specifically evaluated intrapartum care using the national standard. Findings, typically, indicate moderate quality of midwifery care in labor wards and underscore the need for continuous assessments (Moosavisadat et al., 2011; Simbar et al., 2005, 2009). Unexpectedly, despite poor quality of care in some domains, women have reported satisfaction (Simbar et al., 2005).

Given the priority of women's health and the significance of maternal mortality and morbidity rate, as an indicator of community health, this study aimed to assess the implementation rate of maternity care based on the national guideline in selected educational hospitals of Tehran. To do this, we first developed and assessed the psychometric properties of a checklist to audit intrapartum practice based on the national guideline developed by the Iran Ministry of Health (Torkestani et al., 2012), which we then applied in an observational study to assess alignment with the guidelines. Second, we surveyed care providers knowledge and attitudes toward the components of the national guideline.

\section{Method \\ Study Subjects}

This observational study was conducted on 200 pregnant women recruited based on quota sampling method. Quota sampling is a nonprobability equivalent of stratified sampling in which participants are selected from the population in a nonrandom manner. First, the strata and their frequency are identified, and then the required number of participants is recruited by convenience sampling (Tyrer \& Heyman, 2016). Accordingly, first, we identified the annual rate of deliveries in each hospital, and then we allocated a proportion to each setting. Then convenience sampling method was used to recruit the required number of participants from each stratum (hospital). Women with single low-risk pregnancy and uncomplicated vaginal delivery met the inclusion criteria; those who needed emergency cesarean section were excluded from the study. As the aim of the study was to audit 
intrapartum care, women were recruited regardless of their age, nationality, and gestational age.

In addition, 40 female care providers (including midwives, obstetricians, obstetric residents, and medical interns) were asked to participate in the study and to complete a questionnaire about their knowledge and attitudes regarding the National Guideline for Midwifery and Birth Services. Care providers were recruited by quota sampling method. The proportion allocated to each hospital was determined based on the annual rate of vaginal delivery in that hospital (i.e., more professionals were interviewed in hospitals with higher birth rates and larger numbers of personnel).

\section{Data Collection}

Instruments. The tools for data collection were (1) an observational checklist to evaluate intrapartum practice and (2) a questionnaire to assess the knowledge and attitudes of care providers regarding the national guideline.

(1) The observational checklist was completed by a researcher (S.M.) during all three shifts, and the questionnaire was completed by the care providers. The observational checklist was developed based on the National Guidelines for Midwifery and Birth Services created by Iran Ministry of Health in 2012 to ensure standard peripartum care practice (Torkestani et al., 2012). The checklist consisted of nine areas of evaluation including (1) history taking, (2) vital sign assessment, (3) performing Leopold's maneuver, (4) initial assessment, (5) labor care, (6) pain relief methods, (7) labor progress, (8) process of delivery, and (9) postpartum management. The checklist included 121 items (presented in Table 1); each item received a coefficient of 1-3 based on its clinical importance. The items were scored 2 for care provided and 0 for care not provided. Also, there was an option of "not applicable."

Those items with similar content were combined and considered as a single item. For example, the item "pelvic and genital examination" in the domain of initial assessment consisted of several subitems including determination of (1) dilatation, (2) effacement, (3) cervical ripening, (4) fetal station, (5) fetal presentation, (6) membranes status (intact or ruptured), and (7) pelvis size; were separately scored initially; summed with each other; and then considered as a single item. In these cases, the whole item (not the subitems) received the coefficient.

The content validity index was assessed by experts' rating to the relevance of the items. The content and face validity of the checklist were confirmed by 15 midwives, obstetricians, and faculty members of the 
Table 1. Frequency and Compatibility Percentage of Provided Care in Different Intrapartum Domains.

\begin{tabular}{llcc}
\hline & Evaluating Items in Each & $N(\%)$ & Mean Compatibility \\
Domain of Care & Category & $(N 1 / 4200)$ & Percentage
\end{tabular}

I. History taking Asking question on:

88.3

- Gestational age

$200(100)$

- Vaginal bleeding

$200(100)$

- Past medical history $176(88)$

- Drug history $200(100)$

- Surgical history $\quad 145(72.5)$

2. Vital sign

assessment

Assessment of

- Blood pressure $200(100)$

- Temperature $132(66)$

- Respiration 37 (18.5)

- Pulse $43(21.5)$

3. Performing

Leopold's

maneuver

4. Initial assessment

Performing

- First maneuver

$104(52)$

- Second maneuver $26(13)$

- Third maneuver $44(22)$

- Fourth maneuver $0(0)$

Assessment of laboratory

$200(100)$

tests and sonographic

scans

Pelvic and genital

$200(100)$

examination

Fetal heart rate assessment $200(200)$

5. Labor care Explaining care for parturient 23 (11.5)

Asking for permission prior $\quad 54$ (27)

to any intervention

Providing suitable information to patient

$38(19)$

Making effective

$116(58)$

communication

Maintaining patient privacy $\quad 73(36.5)$

Restriction/prohibition of $200(100)$

oral intake

38.5

83.4

64.6 
Table 1. (continued)

\begin{tabular}{|c|c|c|c|}
\hline Domain of Care & $\begin{array}{l}\text { Evaluating Items in Each } \\
\text { Category }\end{array}$ & $\begin{array}{c}N(\%) \\
(N 1 / 4200)\end{array}$ & $\begin{array}{c}\text { Mean Compatibility } \\
\text { Percentage }\end{array}$ \\
\hline & $\begin{array}{l}\text { Support for the mother's } \\
\text { choice of position }\end{array}$ & $138(69)$ & \\
\hline & $\begin{array}{l}\text { Encourage the mother to } \\
\text { walk around freely }\end{array}$ & $46(23)$ & \\
\hline & $\begin{array}{l}\text { Encouraging mother to } \\
\text { empty her bladder }\end{array}$ & $23(11.5)$ & \\
\hline
\end{tabular}

6. Pain relief Use of breathing techniques $73(36.5)$

methods Use of pharmacologic pain $86(43)$ relief

7. Labor progress Appropriate vaginal

$67(33.5) \quad 71.5$

examination

Use of continuous fetal heart 200 (100) monitoring

Vital sign assessment $\quad 162(81)$

8. Process of Encouraging mother to push $200(100)$

89.5

delivery $\quad$ Performing routine $\quad$ I67 (83.5)

episiotomy

Initial newborn assessment $200(100)$

Encouraging initiation of 33 (16.5) breastfeeding

Active management of third 200 (100) stage

9. Postpartum

Vital sign assessment

$162(81)$

89.5 management

Use of uterotonic agents

$200(100)$

Keeping the mother warm $\quad 40(20)$

and stable

Ensuring rooming-in for

14 (7) mother and baby

General assessment of mother

$200(100)$ 
university. Intrarater reliability assessment of the checklist demonstrated a 93 Pearson correlation coefficient.

(2) The questionnaire was designed to assess the knowledge and attitude of care providers regarding the instructions of the national guideline. The tool was divided into three sections of individual, environmental, and occupational parts. Each of the knowledge and attitude sections 
consisted of 10 questions. Knowledge questions were answered by "yes," "no," and "I don't know," which were scored 0-2 based on the accuracy of the responses. Attitude questions were responded by "agreed," "disagreed," and "have no idea" and were scored 0-2 based on the positivity or negativity of the answers.

Items in the environmental and occupational parts were selected based on some intrapartum care practices that were commonly misused according to the available literature and were also major intrapartum challenges in our study. In addition, prior to data collection, the selected items were discussed with a number of care providers through interview and were revised according to their comments. Multiple choice questions were used to inquire on the following issues: (1) having someone to accompany parturient, (2) support for the mother's choice of position, (3) encourage the mother to walk around freely, (4) restriction/prohibition of oral intake, (5) continuous fetal monitoring, (6) use of nonpharmacologic pain relief, and (7) performing routine episiotomy. Total scores for each item were presented as percentage. There were also questions about the demographic characteristics of care providers. We interviewed 10 care providers to check the content validity of the tool. Test-retest reliability assessment of the questionnaire revealed a correlation coefficient of 9 .

Data were collected in four educational hospitals of Tehran (Shahid Akbarabadi, Arash, Mirzakouchak Khan, and Hedayat). Informed verbal consent was obtained from all participants, and the study was approved by the ethics committee of Shahid Beheshti University of Medical Sciences.

\section{Statistical Analysis}

Continuous variables were presented as mean (standard deviation) or frequency (percentage). To analyze the data of the checklist, the scores of each domain were summed with each other, and the final calculated score was divided by the potential highest score of that domain and then the percentage was calculated. The obtained percentage scores for compatibility with the guideline were interpreted as acceptable $(67-100 \%)$, moderate (34-66\%), or not acceptable (0-33\%). To present findings of the questionnaire, the scores given to each option were summed up and then presented as percentage. The Pearson correlation coefficient was applied to express the association between quantitative variables. Significance level was set at $p<.05$. Statistical analysis was performed using SPSS Version 17.0 (Chicago, IL). 


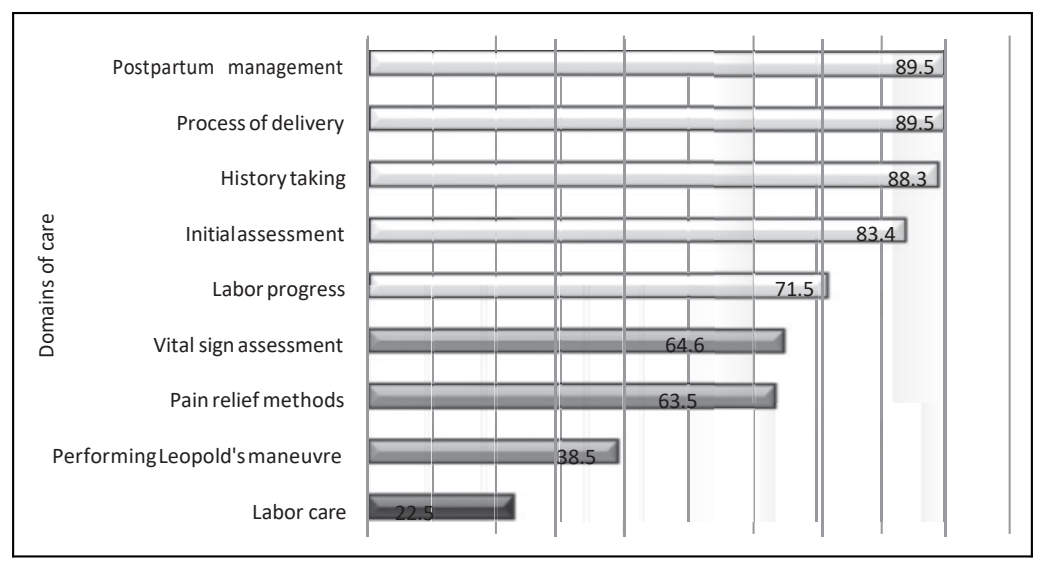

Figure 1. Mean compatibility percentage of intrapartum practice with the national guideline for midwifery and birth services.

\section{Results}

Of a total 200 parturients aged $25.04+7.63$ years, $84 \%$ were in their first or second pregnancy (parity 1-2), $88 \%$ were housewives, and nearly $50 \%$ had completed primary school. Of a total 40 care providers aged $27.12+$ 6 years, $80 \%$ were obstetrics residents, interns, or midwifery students. Twenty-five percent of them had 1-2 years clinical experience, and $17.5 \%$ had experience in educational hospitals.

Table 1 indicates the frequency of each item in different domains. In the domain of history taking, main questions were asked from all participants. In the domain of vital sign assessment, although an item such as blood pressure measurement was performed for all cases, other items such as respiration and pulse rate measurement were assessed only in $18.5 \%$ and $21.5 \%$ of cases, respectively. During labor, women were routinely restricted from oral intake $(100 \%)$ and were asked to undergo perineal shaving $(100 \%)$. Only $23 \%$ of the women were encouraged to walk around freely. Use of breathing techniques, as a pain relief method, was performed for $36.5 \%$ of cases.

Compatibility of intrapartum care with the national guideline was different between domains (Figure 1). The highest scores were related to the domains of "process of delivery" $(89.5 \%)$ and "postpartum management" $(89.5 \%)$; whereas, only $22.5 \%$ of the care provided in the domain of labor care was in alignment with the national guideline (lowest compatibility). 
Overall, the scores in the domains of history taking, initial assessment, and labor progress were acceptable.

The reasons for inappropriate but common intrapartum care were also assessed by the questionnaire. Care providers responded to each question based on their perspectives. Since for each question, one or more options could be chosen, the percentage for some questions could be higher or lower than $100 \%$. Some items were scored 0 , as providers did not perceive them to be an important underlying reason for that specific care. Of a total 40 participants, $27.1 \%$ considered space limitations as the main reason for not having someone to accompany parturient; $24.4 \%$ selected lack of knowledge for not supporting mother's choice of position and $25 \%$ considered crowded wards as the main reason for not encouraging the mother to walk around freely (Table 2).

The knowledge and attitude assessment of care providers regarding the national guideline revealed that $57 \%$ of them had medium knowledge and $45 \%$ had positive attitude toward the guideline. There were significant correlations between knowledge and attitude (Pearson correlation: $r^{1 / 4} .69, p<.001$ ), knowledge and practice (Pearson correlation: $r^{1 / 4} .72$, $p<.03$ ), and knowledge and clinical experience of care provider (Pearson correlation: $r^{1 / 4} .78, p<.01$ ).

\section{Discussion}

In the present study, we audited the compatibility of intrapartum care with the Iranian National Guideline for Midwifery and Birth Services in Tehran, Iran. The guideline consisted of various components depending on different stages of labor and delivery. Our findings showed that the least acceptable practices were performed in the domain of labor care. Over the past decades, the number of women who planned to give birth in hospitals have enormously increased; unfortunately, a simultaneous increase in mistreatments and abusive practices in maternity units has been reported as well (WHO, 2014). High-quality care during labor is crucial in ensuring a safe childbirth and a healthy neonate. The importance of safety and quality care is even more significant for those practices, which directly involve prevention of harm or reduction in adverse maternal outcomes (Shojania, Duncan, McDonald, Wachter, \& Markowitz, 2001). Typically, clinicians should avoid any intervention, unless there is a valid reason to interfere with the physiologic process of birth (Technical Working Group, WHO,1997). In this respect, we discussed some of routine inappropriate but common practices and interventions during childbirth, which are more frequently 
Table 2. Underlying Reasons for Common Inappropriate Intrapartum Practices in the View of 40 Care Providers.

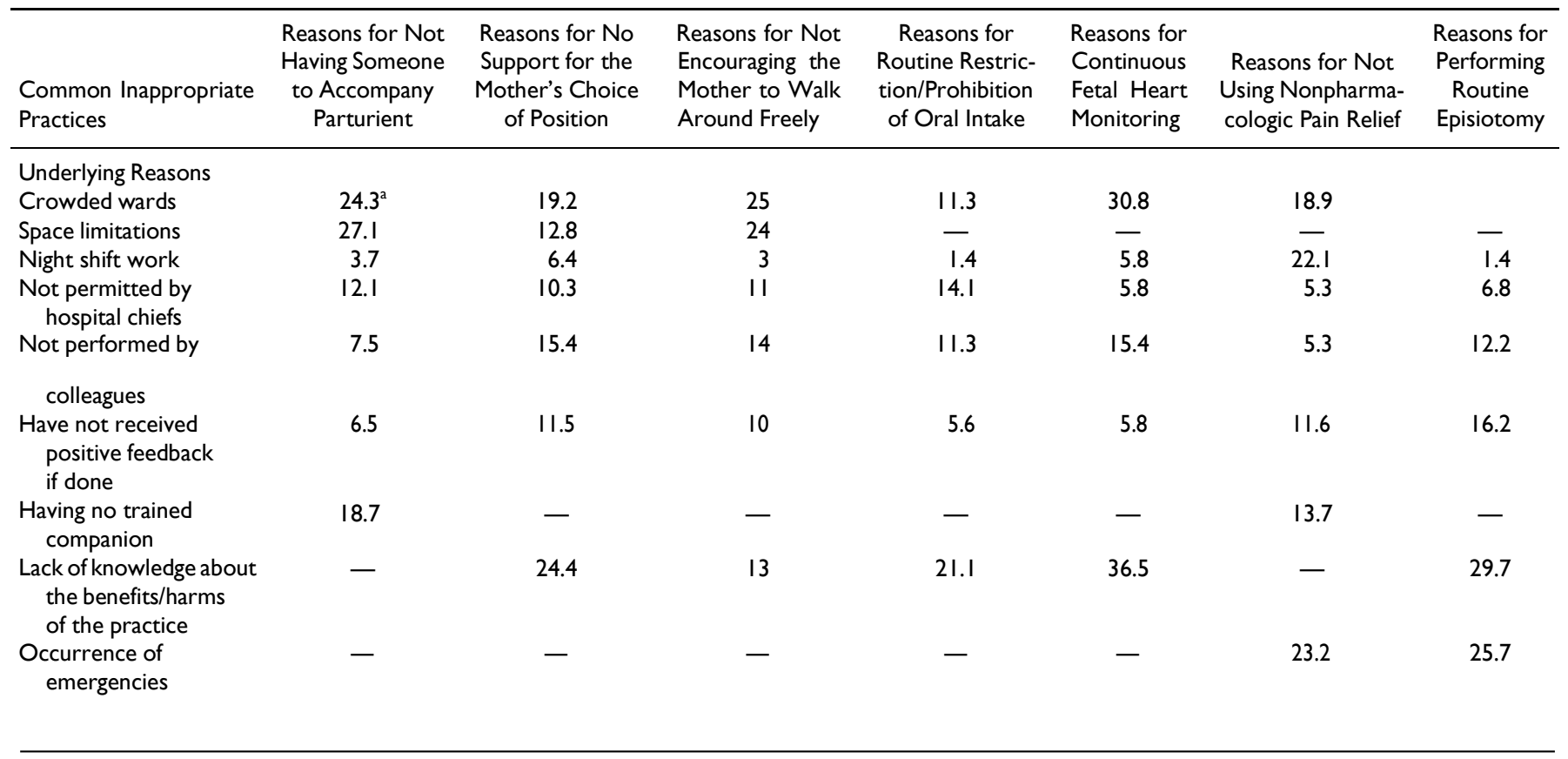

${ }^{\mathrm{a}}$ Data given as percentage. 

reported in the literature; these are either effective or aggressive interventions which, respectively, their underuse or overuse may contribute to maternal complications (Miller et al., 2016). To evaluate the general knowledge and attitude of the care providers, we also performed a survey of which its main findings have been presented.

The latest Cochrane review clearly confirms the importance of walking and choosing upright positions (Lawrence, Lewis, Hofmeyr, \& Styles, 2013 ); however, in our study, only $23 \%$ of mothers were encouraged to do so. Walking can reduce the duration of first stage and does not seem to impose any negative effect on mother and fetus (Lawrence et al., 2013). Based on the responses received from care providers, we recognized that crowded labor wards and space limitations were two underlying factors that precluded midwives to recommend this option to mothers.

Comparable to previous reports (Changaee et al., 2014; Simbar et al., 2009), none of our study participants were permitted to have a companion throughout labor and delivery. In most of Iranian hospitals, fathers are legally prohibited from entering birth rooms; however, due to new regulations, this situation has been improved in the recent years (Mortazavi \& Mirzaii, 2012). Public hospitals are gradually providing new labor--delivery-recovery rooms in delivery wards which makes the presence of husbands convenient (Tribune, 2017). Presence of a companion during labor increases the likelihood of vaginal delivery and can reduce the rate of cesarean section or instrumental deliveries (Hodnett, Gates, Hofmeyr, \& Sakala, 2013).

The majority of care providers in our survey responded that lack of knowledge about the potential benefits/harms of the practice, especially in the case of emergency cesarean section, led them to prohibit parturient from eating or drinking. However, this is in contrast with the current evidence which does not support any benefit/harm attributable to restricting fluid and food intakes (Singata et al., 2013). Therefore, in low-risk pregnancies, there is no justification for prohibition of oral intake.

Our results showed that all women irrespective of their low- or high-risk conditions were assessed by continuous fetal monitoring, a practice that is contradicted by expert bodies (American College of Obstetricians and Gynecologists [ACOG], 2009). Despite benefits of continuous monitoring in pregnancies complicated with preeclamsia, diabetes, or intrauterine growth restriction, other pregnant women can benefit from intermittent auscultation alone (ACOG, 2009). Since the results of this study revealed that the lack of knowledge regarding the benefits/harms of fetal monitoring 
was the main reason for its continuous use in labor, more training courses seem to be beneficial for our care providers.

We also found that routine episiotomy was performed in $83.5 \%$ of the cases, which is far more than the rates reported by other countries (Chalmers, Kaczorowski, O' Brien, \& Royle, 2012; Karacam, Ekmen, \& Calisir, 2012; Li et al., 2015). According to the Cochrane 2017 report, the recommended practice is selective episiotomy, and routine episiotomy should be limited only to high-risk deliveries such as those assisted by vacuum (Jiang et al., 2017). The present study showed low knowledge of the providers about the benefits/harms of episiotomy which might be the key factor for its routine use. However, we think that the educational environment of the hospitals might have exacerbated the condition.

Our results demonstrated that the highest scores were for two domains of "delivery process" and "postpartum management." Assisting parturients in delivery, suctioning, and warming of neonate and placental delivery/ evaluation were all properly performed. Active management of the third stage was followed by oxytocin infusion in $100 \%$ of cases which altogether indicate the considerable attention paid to these vital procedures.

Consistent with previous findings (Simbar et al., 2005, 2009), encouraging mothers to initiate breast-feeding and rooming-in were not fulfilled in $76 \%$ and $85 \%$ of the cases, respectively, which may partly be explained by the insufficient knowledge and neutral attitude of care providers toward skin-toskin contact and early breast-feeding. Besides, while prenatal education of mothers has a key role in successful breast-feeding within the first hour of life (Vieira et al., 2010), many Iranian women still do not attend pregnancy classes, and, therefore, miss the necessary educations in this regard.

Overall, our study is among pioneer researches that have employed national guideline instead of international instructions. However, the study has limitations; we did observations among low-risk pregnancies, and therefore, studies in complicated pregnancies are warranted. Also, the small sample size of 40 care providers may restrict our data generalizability which necessitates further extensive studies to develop more detailed instruments for evaluating the implementation rate of intrapartum care as well as for exploring the reasons of discrepancy between practice and the national guideline.

\section{Conclusion}

We found that care in some intrapartum domains were not in alignment with the national guidelines. Regular training courses and continuing 
education on intrapartum care can empower care providers and improve the quality of care.

\section{Acknowledgment}

The authors acknowledge the study participants and hospital staffs for their assistance in completion of the study.

\section{Declaration of Conflicting Interests}

The author(s) declared no potential conflicts of interest with respect to the research, authorship, and/or publication of this article.

\section{Funding}

The author(s) received no financial support for the research, authorship, and/or publication of this article.

\section{ORCID iD}

Sonia Minooee (D) http://orcid.org/0000-0003-3680-2163

\section{References}

Akbary, M. E. (2005, April). Reduction of maternal mortality rate. Iran-Newspaper, 14, 11.

American College of Obstetricians and Gynecologists. (2009). Intrapartum fetal heart rate monitoring: Nomenclature, interpretation, and general management principles. Obstetrics and Gynaecology, 114, 192-202.

Chalmers, B., Kaczorowski, J., O’Brien, B., \& Royle, C. (2012). Rates of interventions in labor and birth across Canada: Findings of the Canadian maternity experiences survey. Birth, 39, 203-210.

Changaee, F., Simbar, M., Irajpour, A., \& Akbari, S. (2014). Quality assessment of peripartum care. Iranian Red Crescent Medical Journal, 16, e9069.

Downe, S., Gyte, G. M., Dahlen, H. G., \& Singata, M. (2013). Routine vaginal examinations for assessing progress of labour to improve outcomes for women and babies at term. Cochrane database of Systematic Reviews, 7, CD010088.

Ganji, Z. (2014). Evaluation of midwifery care quality and satisfaction of its in labor and delivery units in kashan university of medical sciences hospitals in 2011. Journal of Urmia Nursing And Midwifery Faculty, 12, 858-865.

Hodnett, E. D., Gates, S., Hofmeyr, G. J., \& Sakala, C. (2013). Continuous support for women during childbirth. Cochrane Database of Systematic Reviews, 7, CD003766. 
Jiang, H., Qian, X., Carroli, G., \& Garner, P. (2017). Selective versus routine use of episiotomy for vaginal birth. Cochrane Database of Systematic Reviews, 2, CD000081.

Karacam, Z., Ekmen, H., \& Calisir, H. (2012). The use of perineal massage in the second stage of labor and follow-up of postpartum perineal outcomes. Health Care for Women International, 33, 697-718.

Lawrence, A., Lewis, L., Hofmeyr, G. J., \& Styles, C. (2013). Maternal positions and mobility during first stage labour. Cochrane Database of Systematic Reviews, 10, CD003934.

Li, Y., Townend, J., Rowe, R., Brocklehurst, P., Knight, M., Linsell, L., ... Hollowell, J. (2015). Perinatal and maternal outcomes in planned home and obstetric unit births in women at 'higher risk' of complications: Secondary analysis of the birthplace national prospective cohort study. British Journal of Obstetrics and Gynaecology, 122, 741-753.

Lothian, J. A. (2009). Safe, healthy birth: What every pregnant woman needs to know. Journal of Perinatal Education, 18, 48-54.

Miller, S., Abalos, E., Chamillard, M., Ciapponi, A., Colaci, D., Comandé, D., ... Langer, A. (2016). Beyond too little, too late and too much, too soon: A pathway towards evidence-based, respectful maternity care worldwide. The Lancet, 388, 2176-2192.

Moosavisadat, S. M., Lamyian, M., Parsap, S., \& Hajizadeh, E. (2011). Comparison of maternity care quality in teaching and non-teaching hospitals in Khorram Abad, Islamic republic of Iran. Eastern Mediterranean Health Journal, 17, 638-645.

Mortazavi, F., \& Mirzaii, K. (2012). Concerns and expectations towards husband's involvement in prenatal and intrapartum cares: A qualitative study. Payesh, 11, 51-63.

Shojania, K. G., Duncan, B. W., McDonald, K. M., Wachter, R. M., \& Markowitz, A. J. (2001). Making health care safer: A critical analysis of patient safety practices. Evidence Report/Technology Assessment (Summ), 43, 668.

Simbar, M., Dibazari, Z. A., Saeidi, J. A., \& Alavi Majd, H. (2005). Assessment of quality of care in postpartum wards of Shaheed Beheshti medical science university hospitals, 2004. International Journal of Health Care Quality Assurance Incorporating Leadership in Health Services, 18, 333-342.

Simbar, M., Ghafari, F., Tork Zahrani, S., \& Alavi Majd, H. (2009). Assessment of quality of midwifery care in labour and delivery wards of selected Kordestan medical science university hospitals. International Journal of Health Care Quality Assurance, 22, 266-277.

Simbar, M., Nahidi, F., Dolatian, M., \& Akbarzadeh, A. (2012). Assessment of quality of prenatal care in Shahid Beheshti medical science university centers. International Journal of Health Care Quality Assurance, 25, 166-176. 
Singata, M., Tranmer, J., \& Gyte, G. M. (2013). Restricting oral fluid and food intake during labour. Cochrane Database of Systematic Reviews, 8, CD003930.

Torkestani, F., Abedini, M., Radpouyan, L., Rahimi, Ghasbeh, S., Hadipour, Jahromi, L., Bakhshandeh, M., ... Emami, Afshar, N. (2012). National guideline of midwifery and birth services (3rd Revision) [Internet] Tehran: Iran Ministry of Health, 2012. Retrieved from http://vc-trethment.kums.ac.ir/kums_content/ media/image/2016/10/92630_orig.pdf

Tribune, Financial. (2017). LDR Rooms Encourage Natural Childbirth. Retrieved from https://financialtribune.com/articles/people/63232/ldr-rooms-encouragenatural-childbirth (accessed 14 May 2018)

Tyrer, S., \& Heyman, B. (2016). Sampling in epidemiological research: Issues, hazards and pitfalls. BJPsych Bulletin, 40, 57-60.

United Nations Population Fund. (2012). Maternal deaths halved in 20 years, but faster progress needed. Press release, 16 May 2012. Retrieved from https://www. unfpa.org/press/maternal-deaths-halved-20-years-faster-progress-needed (accessed 14 May 2018).

Vieira, T. O., Vieira, G. O., Giugliani, E. R. J., Mendes, C. M. C., Martins, C. C., \& Silva, L. R. (2010). Determinants of breastfeeding initiation within the first hour of life in a Brazilian population: Cross-sectional study. BMC Public Health, 10, 760.

World Health Organization UNICEF World Bank United Nations Population Fund. Maternal mortality in 2005: estimates developed by WHO, UNICEF, UNFPA, and The World Bank. Geneva: World Health Organization; 2007. Retrieved from http://www.who.int/iris/handle/10665/43807\#http://www.who.int/iris/handle/ $10665 / 43807$.

WHO, UNICEF, UNFPA, The World Bank. Trends in maternal mortality 19902008: estimates developed by WHO, UNICEF, UNFPA and The World Bank Geneva: World Health Organization; 2010. Retrieved from: www.who.int/repro ductivehealth/publications/monitoring/9789241500265/en/index.html (accessed 14 May 2018)

World Health Organization. WHO Statement on the Prevention and Elimination of Disrespect and Abuse during Facility-based Childbirth [Internet]. Geneva: World Health Organization, 2014. Retrieved from http://apps.who.int/iris/bit stream/10665/134588/1/WHO_RHR_14.23_eng.pdf?ua=1\&ua=1 (accessed 14 May 2018)

Technical Working Group, World Health Organization. (1997). Care in normal birth: a practical guide. Birth, 24(2),121-3. 